\title{
Remeasurement at High Resolving Power in Fourier Transform Ion Cyclotron Resonance Mass Spectrometry
}

\author{
Victoria L. Campbell, Ziqiang Guan, and David A. Laude \\ Department of Chemistry and Biochemistry, University of Texas at Austin, Austin, Texas, USA
}

\begin{abstract}
The Fourier transform ion cyclotron resonance mass spectrometry remeasurement experiment is demonstrated and evaluated under high resolution conditions. Signal-to-noise enhancement is observed for isotopically resolved bovine insulin peaks at a resolution of $\sim$ 31,000 (full width at half height). The experiment is sensitive to space-charge effects and resultant changes in scan-to-scan signal-to-noise and resolution. Coulombic repulsion in the ion cloud during the high resolution remeasurement experiment can cause the cyclotron frequency to shift through the duration of the experiment, which results in broadened peak shapes when individual remeasurement spectra are coadded. By either reducing the number of ions in the cell or allowing the ion cloud to diffuse during the lifetime of the experiment, high resolution remeasurement spectra can be coadded without peak broadening or degradation of signal-to-noise ratio. (J Am Soc Mass Spectrom 1995, 6, 564-570)
\end{abstract}

$\mathrm{F}$ ourier transform ion cyclotron resonance (FTICR) mass spectrometry is gaining popularity as a versatile technique for the study of biomolecular ions produced by electrospray ionization (ESI) [1-4]. The characteristics of high performance FTICR that make it especially suited to the analysis of large, multiply charge electrosprayed ions include high resolving power, mass accuracy, and sensitivity. This high resolution capability makes it possible to determine mass assignments from isotope pattern and spacing for multiply charged product ions formed from either ion-molecule reactions or ion dissociation techniques. For example, Senko et al. [5] obtained sequence information for carbonic anhydrase by using a high performance ESI-FTICR mass spectrometer to resolve the carbon isotopes of fragment ions.

In contrast to other mass spectrometric techniques, FTICR is capable of continuously monitoring a single ion population because the detection process is not destructive. If after the ion detection event the ions lose sufficient kinetic energy via a homogeneous relaxation process to return to their original low energy cyclotron radius [6], they can be remeasured during subsequent reexcitation and detection. Efficient ion remeasurement can provide several advantages, which include signal-to-noise enhancement, lower detection limits, continuous ion monitoring, and true $\mathrm{MS}^{\mathrm{n}}$. In 1990, Williams et al. [7] used plasma and laser desorp-

Address reprint requests to Professor David A. Laude, Jr., Department of Chemistry and Biochemistry, University of Texas at Austin. Austin, TX 78712. tion to first demonstrate remeasurement, and they showed that simple collisional cooling with low mass background neutrals is increasingly effective in reduction of the cyclotron radius as the size of ions increases. Then, in 1993, Guan et al. [3] applied these principles to broadband remeasurement of electrosprayed proteins as large as $132,000 \mathrm{u}$ and found that unit remeasurement efficiency was easily achieved for these compounds even over several hundred scans of the same ion population while simultaneously detecting the entire charge state distribution, which covered a $m / z$ 1800-3200 region. More recently, Guan $[8,9]$ has taken advantage of the efficiency of the FTICR remeasurement experiment to observe the ion-molecule reactions of a single ion population. Continuous monitoring of gas phase ion chemistry between protein ions and diethylamine allowed time-resolved adduct formation and charge stripping products to be observed $[8,9]$. Further study of the collisionally cooled remeasurement process revealed that stringent requirements on both cell geometry and ion cyclotron radius are necessary to obtain unit remeasurement efficiencies [10].

Remeasurement performed in conjunction with quadrupolar excitation $(\mathrm{QE})$ remeasurement has been shown by Speir et al. [11] and C. L. Hendrickson and D. A. Laude [12] to be effective and requires fewer constraints on ion cyclotron radius and cell geometry. However, neither of these demonstrates the ability to do broadband remeasurement; efficient remeasurement is obtained only at a single mass-to-charge ratio value. The methodology for broadband $\mathrm{QE}$ is currently being developed by Guan and Marshall [13, 14]; a 
broadband detection range of $m / z \quad 1500-2500$ is the best observed [15]. Nevertheless, broadband QE remeasurement has not been demonstrated to date.

Similarly, high resolution remeasurement has never been demonstrated with or without the use of QE. Given the merits of both high performance ESI-FTICR and the FTICR remeasurement experiment, it is the goal of this work to demonstrate and evaluate the combination of high mass resolution and remeasurement. Unexpectedly, difficulties were encountered in initial attempts to establish conditions for the high resolution remeasurement experiment. The scan-toscan shift of isotopic peaks to increased cyclotron frequency resulted in spectral line broadening, which delayed the onset of isotopic resolution in the remeasurement mode. These anomalies were attributed to changes in the ion density induced by Coulomb broadening. Once the space-charge in the cell was reduced, remeasurement of isotopically resolved charge states was achieved.

\section{Experimental}

All data were obtained with an FTICR mass spectrometer coupled to an electrospray ionization source. The spectrometer included a 6-in.-diameter bore, a 3.0-T superconducting magnet, and four concentric vacuum chambers of increasing diameter that extended into the high-field region of the magnet. A mechanical shutter was used to gate the flow of neutrals into the cell region [16, 17]. The cell region conductance limit was electrically isolated to gate the injection of ions into a 2-in.-diameter open cylindrical cell of 2:1 aspect ratio. Ions were efficiently injected and trapped in the cell only when the potential of the trap plates matched the ion kinetic energy per unit charge [18-20]. Electrospray ionization was performed by application of a potential of $3.7 \mathrm{kV}$ to the $100-\mu \mathrm{m}$-i.d. blunt-end syringe needle. The syringe needle was positioned $6 \mathrm{~mm}$ from the $500-\mu \mathrm{m}-\mathrm{i} . \mathrm{d}_{.}, 20-\mathrm{cm}$ desolvating capillary, which was biased to $330 \mathrm{~V}$ and resistively heated to $\sim 150^{\circ} \mathrm{C}$ by an applied current of $2.2 \mathrm{~A}$. The skimmer cone was typically biased between +5 and $+15 \mathrm{~V}$ to obtain desired electrospray ion kinetic energies in the direction of the cell.

\section{Electrospray Parameters}

Solutions were pumped to the syringe needle through a 22-gauge Teflon tube via a Harvard Apparatus (South Natick, MA) 22-gauge syringe infusion pump that delivered a flow rate of $1-4 \mu \mathrm{L} / \mathrm{min}$. Solutions were prepared by dissolving the sample in an aqueous solution of acetic acid and then dilution with methanol to achieve a final solution concentration of $5-10 \mathrm{pmol} / \mu \mathrm{L}$ with a volume ratio of 30:68:2 water:methanol:acetic acid. Horse heart myoglobin (molecular weight 16,951) and bovine insulin (molecular weight 5734) were used as obtained from Sigma Chemical Co. (St. Louis, MO).

\section{Data Acquisition}

Data acquisition and processing were performed with an Extrel Odyssey Data Station (Extrel FTMS, Inc., Madison, WI) that ran software version 2.0. A two-sequence script was written for the remeasurement experiment. The FTICR remeasurement script had the form $\left\{S_{0}-P V_{1}-I-S_{c}-D_{z}-\left[E x-A-P V_{2}-D_{x y}\right]_{n}-Q\right\}$ with the individual events as defined below. The first sequence was initiated by opening the mechanical shutter $S_{0}$ to allow the ion-neutral beam into the lower pressure regions of the vacuum system. The opening of the shutter was immediately followed by a pulse of nitrogen gas, $\mathrm{PV}_{1}$, that increased the pressure to approximately $3 \times 10^{-5}$ torr to facilitate the accumulated trapping of ions, The duration of the ion injection period I was characterized by the conductance limit being held at ground potential to allow the flux of ions into the cell: $\mathrm{A}+10-\mathrm{V}$ potential was applied to the cell region conductance limit before and after the injection event while the shutter was open to repel the ion beam away from the cell. After the ionization event, the shutter was closed, $S_{c}$, followed by a 10-30-s delay $D_{z}$ to collisionally cool the axial motion of the ions. The second sequence controlled multiple applications $n$ of the four-event remeasurement cycle. Excitation was performed via a linear broadband excitation sweep Ex [21, 22]. The magnitude of the excitation was varied systematically to optimize the remeasurement efficiency of the experiment. Broadband mass spectra were acquired, $\mathrm{A}$, in direct mode with sufficient data points for the massto-charge ratio range of interest to resolve charge states; mass spectra were acquired in the heterodyne mode within the mass-to-charge ratio range of interest to resolve isotope peaks. After data acquisition, nitrogen gas was pulsed into the cell region, $\mathrm{PV}_{2}$ to increase the pressure to approximately $5 \times 10^{-6}$ torr, followed by a cooling delay $D_{x y}$ of about $5 \mathrm{~s}$ to facilitate the collisional relaxation of the ions back to their preexcitation radius at the center of the cell before the initiation of the subsequent remeasurement cycle. The pulse sequence was terminated by a single quench event $Q$ that purged all charged particles from the cell.

During the events of the first remeasurement sequence the trap potentials were typically tuned to a potential between 7.5-9.5 V. However, after the axial cooling delay $D_{z}$, the trap potentials of the events in the second remeasurement sequence were set to less than $1.0 \mathrm{~V}$.

\section{Results and Discussion}

\section{Successful Remeasurement under High Resolution Conditions}

The ability to remeasure electrosprayed protein ions with high efficiency under low resolution conditions is well established. For example, as many as 250 consecutive remeasurements of an initial population of bovine 
albumin dimer ions were made with little apparent loss in ion number [3]. However, these experiments were performed under data acquisition conditions that yielded data-point-limited mass resolution values of about $m / \Delta m=1000$, which are sufficient only to resolve individual charge states. Although the advantages of continuous detection of a single ion population for an extended period of time only have begun to be exploited in the literature, no work has been published that demonstrates the ability to remeasure ions under high resolution conditions. Here we present a demonstration and evaluation of the high resolution remeasurement experiment. A single population of bovine insulin ions was remeasured for 30 consecutive scans. Figure 1 shows that the expected theoretical signal-to-noise ratio enhancement is achieved while isotopically resolved peaks are maintained with an average resolution of $\sim 31,000$ (full width at half height). To maintain isotopic resolution while obtaining unit remeasurement efficiencies, several parameters were modified from the low resolution experiment. From an earlier study [10], we determined that

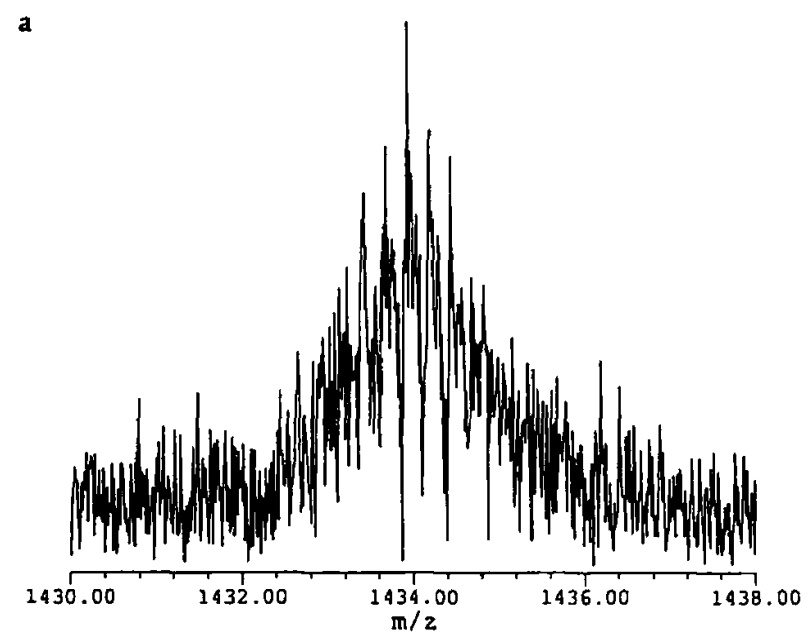

b

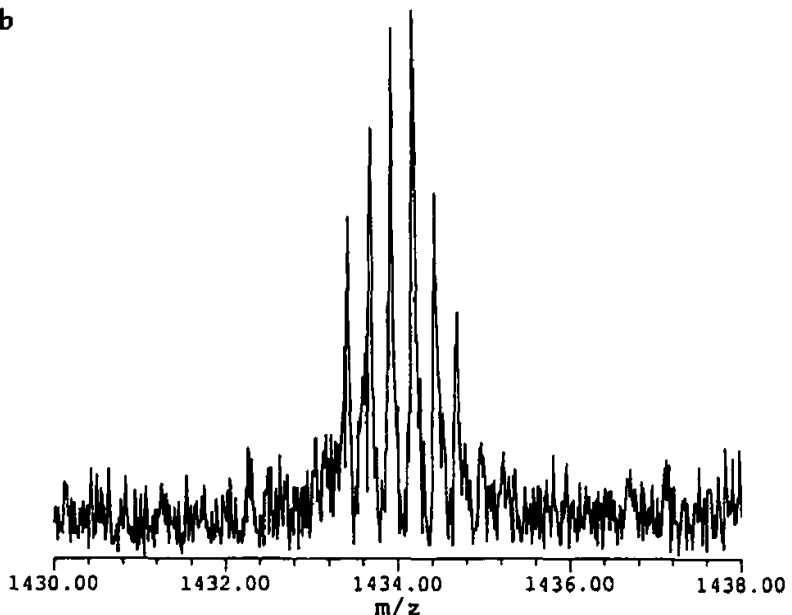

Figure 1. Comparison of unapodized ESI-FTICR high resolution remeasurement spectra of the +4 charge state of insulin obtained by using an applied trap potential of $0.1 \mathrm{~V}$ for (a) 1 and (b) 30 coadded remeasurement scans. small ion radii, typically $\sim 18 \%$ of the cell radius, are required in the open cylindrical cell to obtain unit remeasurement efficiency. Therefore, an attenuation of $20 \mathrm{~dB}$ was applied to a linear broadband excitation at a sweep rate of $200 \mathrm{~Hz} / \mu$ s from $\mathrm{m} / z$ 1000-2000. Also, the cooling delay between remeasurements was increased from 2 to $5 \mathrm{~s}$ to "relax" the ion cloud to the smallest possible phase-space volume.

\section{Frequency Shifts in Remeasurement Spectra}

Initial efforts to obtain signal-to-noise enhancement while retaining isotopically resolved peaks were not successful. Peak shifting observed in the initial experiments was sufficient to cause deterioration of the isotopic pattern when consecutive high resolution remeasurement spectra were coadded. A comparison of low and high resolution remeasurement spectra in Figure 2 indicates the disparity between the two experiments. Figure 2a presents low resolution remeasurement spectra for the +17 charge state of horse heart myoglobin for 1 and 16 coadded scans, respectively. The improvement in signal-to-noise ratio is evident. It is also important to notice the modest downward shift in massto-charge ratio that corresponds to an increase in the effective cyclotron frequency with increasing remeasurement number. However, because there are insufficient data points to resolve the fine structure of the peak, the overall frequency shift of a few hundred hertz is inadequate to alter the basic structure of a peak that is several thousand hertz wide at the baseline.

In contrast, the high resolution coaddition of 1 and 16 remeasured scans of the +4 charge state of insulin in Figure $2 \mathrm{~b}$ exhibits a deterioration of both mass resolution and spectral signal-to-noise ratio. In this example as well, a few hundred hertz increase in effective cyclotron frequency is evident over the course of the remeasurement experiment. However, with peak widths at the half height of only a few hertz, the frequency shift is sufficient to lose resolution of isotopes within the charge state. The data in Figure 2 indicate that frequency shift during consecutive high resolution remeasurements can be a significant problem. To reduce or eliminate the deleterious effects of frequency shift on the high resolution remeasurement experiment, the underlying causes of the shift were examined.

A more quantitative evaluation of the cyclotron frequency shift as a function of remeasurement number was conducted. Shown in Figure 3 are the scan-toscan cyclotron frequencies for three isotopes of the +4 charge state of insulin over the course of nearly 60 remeasurements. The profiles indicate that an increase in frequency of about $15 \mathrm{~Hz}$ occurs. The greatest rate of change in frequency occurs in the initial remeasurements, whereas the rate of change is greatly reduced at later remeasurement times. Importantly, there appears to be no fluctuation of the frequency spacing between 
a
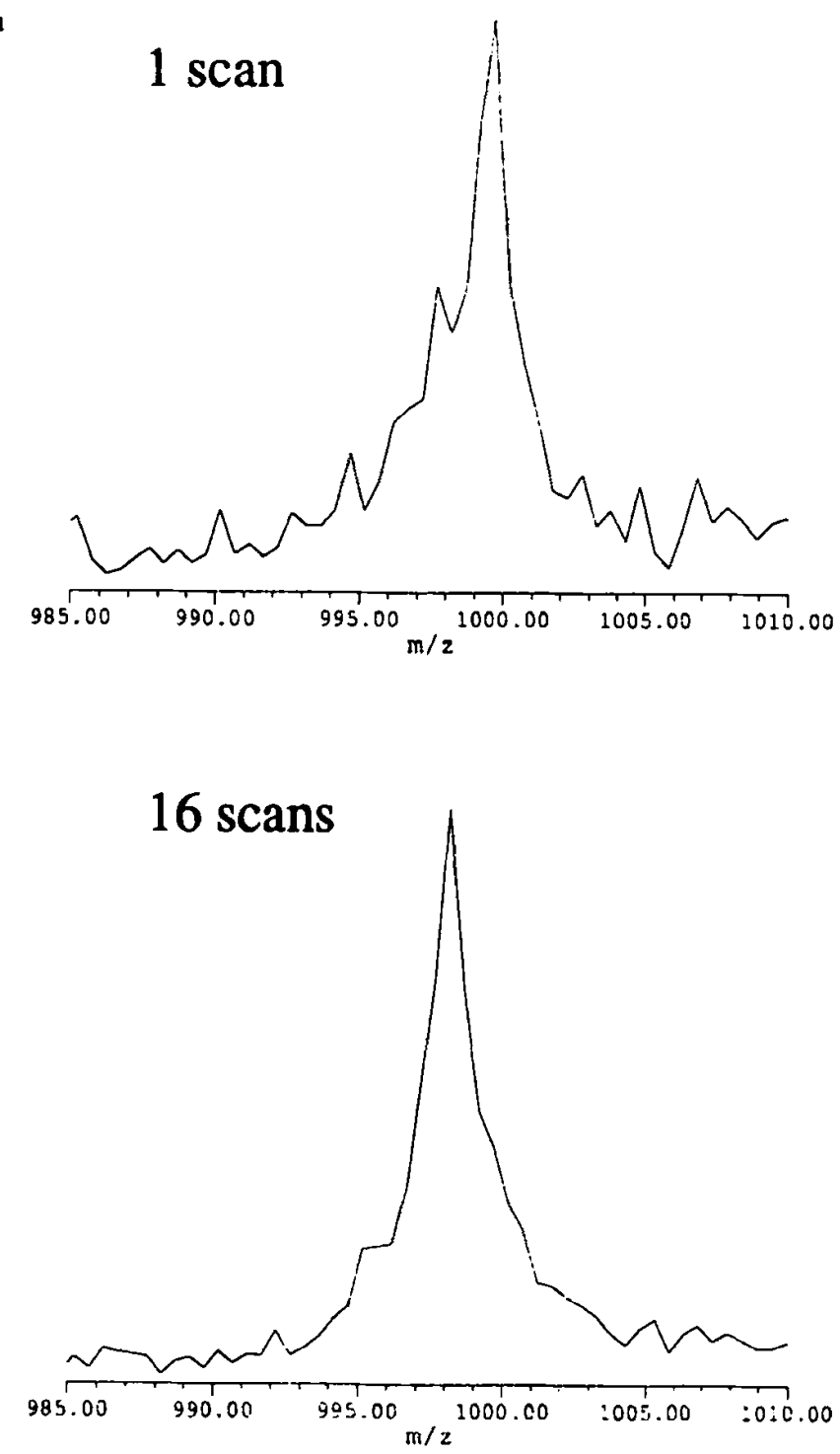

b
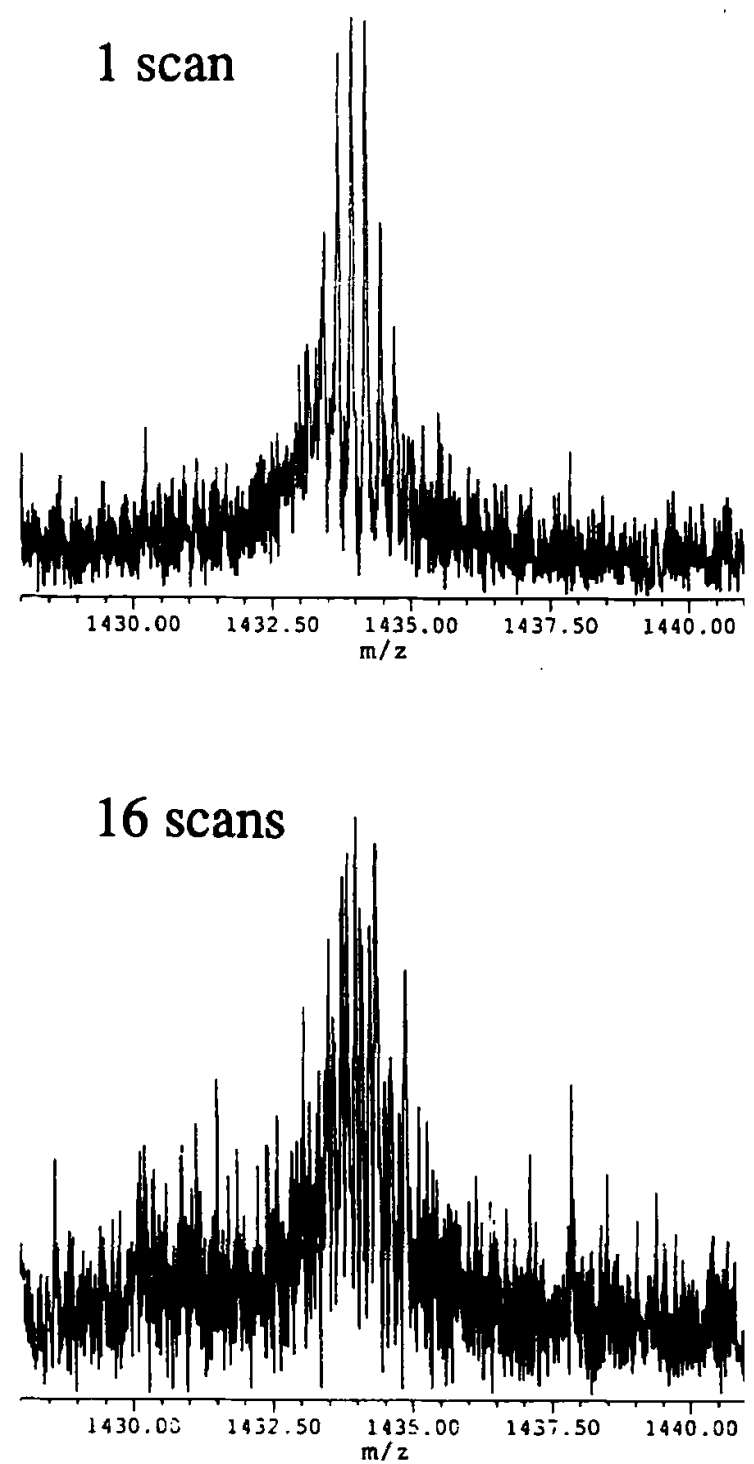

Figure 2. Comparison of unapodized ESI-FTICR remeasurement spectra: (a) Low resolution spectra of the +17 charge state of horse heart myoglobin for 1 scan and 16 coadded remeasurement scans and (b) high resolution spectra of the +4 charge state of insulin derived from 1 scan and 16 coadded remeasurement scans. Low resolution spectra were acquired at a 1.0-V applied trap potential with $64 \mathrm{~K}$ data points in a $1-\mathrm{MHz}$ bandwidth that yielded a data-point-limited mass resolution of $\sim 900$ full width at half height. High resolution spectra were acquired at a $0.8-\mathrm{V}$ applied trap potential in the heterodyne mode with $4 \mathrm{~K}$ data acquired in a $10-\mathrm{kHz}$ bandwidth that yielded a pressure-limited mass resolution of $\sim 26,000$ full width at half height.

isotopes; instead, a systematic shift in the cyclotron frequency of the isotope envelope from measurement to measurement is revealed.

\section{Space-Charge Contribution to Frequency Shift}

An effort was made next to determine the source of the frequency shift. It is well known that ion cyclotron frequency is a function of both magnetic field and various contributions to the radial electric field in the cell. The effective cyclotron frequency can be calculated by using the equation

$$
\omega_{\text {eff }}=q B / m-2 \alpha V_{\mathrm{T}} / a^{2} B-q \rho G_{\mathrm{i}} / \epsilon_{0} B
$$

where $\omega_{\text {eff }}$ is the effective cyclotron frequency, $q$ is ion charge, $B$ is magnetic field strength, $m$ is ion mass, $\alpha$ is a trap geometry constant, $V_{\mathrm{T}}$ is trap voltage, $a$ is trap width between detector electrodes, $\rho$ is charge density, $G_{\mathrm{j}}$ is an ion cloud geometry factor that is trap geometry dependent, and $\epsilon_{0}$ is the permittivity of vacuum $[23,24]$. The first term in eq 1 represents the unperturbed cyclotron frequency, the second term originates from the radial electric field created by the dc trapping potential and cell geometry, and the third term originates from the radial electric field generated by the Coulombic repulsion of the ion cloud. From this equation, the magnitude of the radial electric field is observed to increase as either trap voltage or charge density increases. Furthermore, in most trapped-ion 


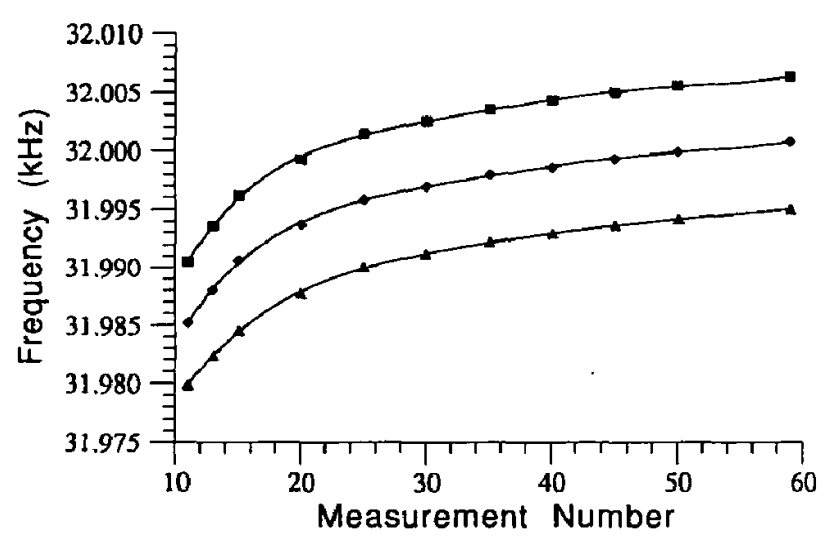

Figure 3. Measured cyclotron frequency of three successive carbon- 13 isotope abundances within the +4 charge state of insulin as a function of measurement number obtained by using an applied trap potential of $0.4 \mathrm{~V}$ during remeasurement: $(\boldsymbol{D})$, $m_{1} / z ;(\diamond),\left(m_{1}+1 u\right) / z ;(\iota),\left(m_{1}+2 u\right) / z$.

cells the magnitude of the radial electric field from the trap potential increases as the ion cloud is accelerated radially to the cell perimeter.

Three scenarios are now considered to explain the frequency shifts that occur in the remeasurement experiment. First, if the radial electric field magnitude is a constant nonzero value because trap potential, ion radius, and charge density all remain constant from scan to scan, the measured cyclotron frequency will remain constant although smaller than that of the unperturbed cyclotron frequency. This is the ideal case and would simplify the effort to obtain high resolution remeasurement spectra. However, the data in Figure 3 indicate that the cyclotron frequency does not remain constant, but shifts over the course of several remeasurements.

A second scenario to explain the frequency shift considers the changing position of the ion cloud in the trapped-ion cell. We have mentioned previously that effective remeasurement requires that ions be retained in a region of the trapped-ion cell with minimal radial electric field. If this does not occur, for example due to a scan-to-scan increase in magnetron radius or inadequate cooling of the ion cloud cyclotron motion, then the ion cloud will experience an ever-increasing radial electric field. As the radial electric field magnitude increases with time, the cyclotron frequency should decrease. However, the data in Figure 3 verify that this does not occur, which refutes the argument for trappotential-related frequency shifts. From an experimental perspective it would seem unlikely also that an ever-increasing ion cloud radius would be responsible for the frequency shift because this would indicate that performance in the remeasurement experiment would degrade with time.

Any plausible explanation for the frequency shift requires the effective radial electric field to decrease over the course of the remeasurement experiment. In this case, the focus in eq 1 should be on the term that describes charge density effects. As the last term indicates, if the magnitude of Coulomb effects decreases during the experiment-either because of ion loss or a reduction in ion cloud density - then the effective cyclotron frequency will increase because the radial electric field decreases. This scenario is consistent with the data in Figure 3.

To test the hypothesis that space-charge effects are responsible for the difficulties in obtaining high resolution remeasurement spectra, remeasurement data were evaluated from scan-to-scan by using several different trap potentials. As is shown in Figure 4, the shape of the frequency shift profile and the onset and duration of the remeasurement experiment are strongly dependent upon trap potential. However, because an increase in the trap potential also increases the rate and magnitude of the positive frequency shift, the applied trap potential must have an indirect effect on the source of the frequency shift. The likely explanation is that the changing trap potential alters the well depth and consequently is an important factor in ion density determination. Specifically, the ion cloud charge density will increase not only because a deeper well will hold a greater number of ions, but also because the axial extent of the ion cloud will decrease as the depth of the trapping well increases. The greater initial charge density for the $0.8-\mathrm{V}$ trap potential in Figure 4 is responsible for the greatest difference between the initial magnitude of the space-charge radial electric field and the radial electric field after the space-charge is alleviated due to ion loss. If ion loss does occur at the larger trap potential, then the magnitude of the frequency shift will increase with applied trap potential. In contrast, for the $0.1-\mathrm{V}$ applied trap potential profile in Figure 4, the magnitude of the radial electric field remains relatively constant, which indicates that a

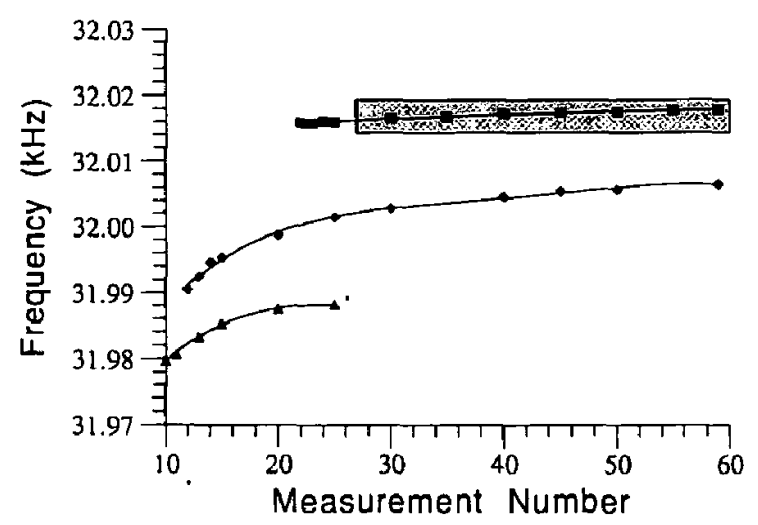

Figure 4. Effective cyclotion frequency of a single carbon-13 isotope in the +4 charge state of insulin as a function of remeasurement number. Profiles were acquired at applied trap potentials during remeasurement of $0.1 \mathrm{~V}(\boldsymbol{\Delta}), 0.4 \mathrm{~V}(\diamond), 0.8 \mathrm{~V}(\Delta)$. Data points are included only for those remeasurement spectra in which all isotopes in the charge state are resolved and observed. The shaded region indicates minimized frequency shift conditions that permit consecutive high resolution remeasurement scans to be successfully coadded. 
fixed ion density is retained throughout the remeasurement experiment.

In support of ion-density-induced frequency shift, the high resolution remeasurement data were replotted in Figure 5 with scan-to-scan signal magnitudes presented for different trap potentials. The profiles indicate that at higher trap potentials there is greater variation in scan-to-scan signal-to-noise ratio, with the early optimum in signal magnitude quickly reduced by subsequent scans. In contrast, the $0.1-\mathrm{V}$ trap potential case exhibits fairly constant signal magnitude throughout the experiment. An explanation for this is that space-charge effects in the early scans promote peak broadening, which consequently reduces peak height. However, as the high resolution remeasurement experiment continues, space-charge density gradually diminishes, which causes mass resolution and peak height to increase. Interestingly, the rate at which overall spectral quality improves is greater at higher trap potential. For example, the first observation of isotopically resolved spectra for the data in Figures 4 and 5 occurs at only eight remeasurement scans for a $0.8-\mathrm{V}$ applied trap potential, but at nearly 20 remeasurement scans for the $0.1-\mathrm{V}$ applied trap potential. One explanation is that radial ion dispersion that leads to a reduction in ion density is proportionally greater at larger trapping potentials [25]. However, this is also a mechanism by which ion loss occurs, which reduces the lifetime of the remeasurement experiment. Thus, the rapid emergence of isotopically resolved peaks that correspond to an optimum ion density is quickly followed by a rapid decay in total ion signal. In contrast, for lower trap potentials, the reduction in ion density necessary to improve mass resolution and signal intensity occurs at a much slower rate, and the rate of ion loss is slower as well. The result is that an optimum in ion density and number for both high mass resolution and signal magnitude is retained for numerous remeasurement scans. The data

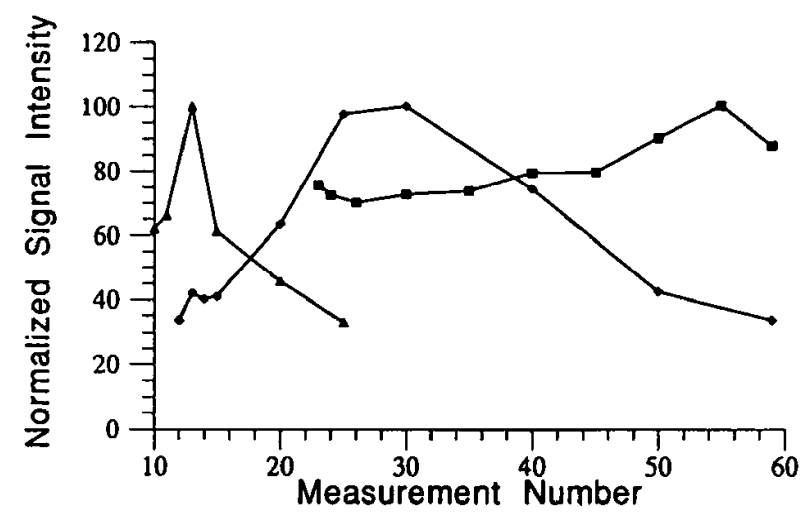

Figure 5. Normalized signal intensity of bovine insulin as a function of measurement number and applied trap potential during remeasurement. Profiles were acquired at applied trap potentials during remeasurement of $0.1 \mathrm{~V}(\boldsymbol{\nabla}), 0.4 \mathrm{~V}(\bullet), 0.8 \mathrm{~V}$ (ム). Data points are included only for those remeasurement spectra in which all isotopes in the charge state are resolved and observed. in Figures 4 and 5 indicate that if sufficient care is taken to define the size and density of the initial ion population, it should be possible to directly coadd consecutive measurements as shown in Figure 1.

\section{Delayed Onset of Isotopic Resolution}

As final verification that space-charge effects play an important role in the high resolution remeasurement experiment, an anomaly observed throughout our efforts to collect such data is presented. As would be expected, if care is not taken to minimize space-charge effects from the initial ion population in the trapped ion cell, then mass resolution is severely degraded. To demonstrate this effect, an excessive number of electrosprayed protein ions were injected into the cell. As shown in Figure 6a, if a typical single scan pulse
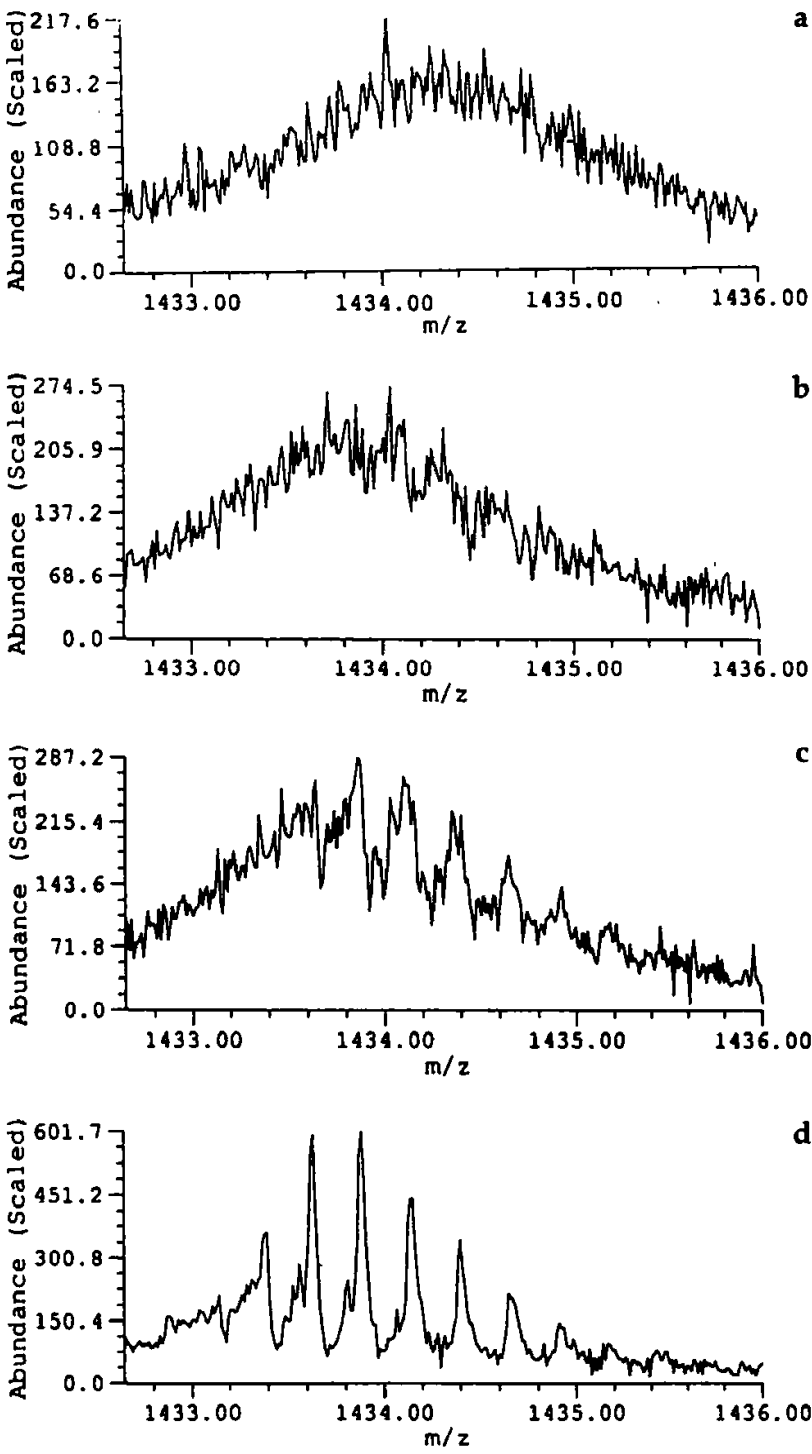

Figure 6. Unapodized ESI-FTICR remeasurement spectra of bovine insulin by using an applied trap potential of $0.4 \mathrm{~V}$ during remeasurement: (a) 8th measurement, (b) 13th measurement, (c) 17 th measurement, and (d) 25th measurement. A long injection time was used to maximize the number of ions in the cell at the beginning of the remeasurement sequence. 
sequence is then executed to detect the ion population, very poor resolution and signal-to-noise ratio are observed in the spectrum because only charge states are resolved. As the experiment progresses a sufficient number of ions are redistributed in the cell or lost until an optimum ion population is achieved for detection. In this case, after 17 remeasurements the first evidence of isotope resolution is observed and by the 25 th scan a mass resolution of nearly 36,000 is sufficient to yield the desired spectrum.

\section{Conclusion}

The FTICR remeasurement experiment offers unique opportunities for signal-to-noise enhancement and monitoring ion-molecule reactions. Under appropriate conditions, high resolution can be successfully combined with efficient remeasurement. However, the high resolution remeasurement experiment is sensitive to space-charge effects, and scan-to-scan changes in signal-to-noise ratio and mass resolution during the remeasurement experiment are common. Nevertheless, space-charge effects can be eliminated effectively by reduction of the number of ions trapped in the cell through the use of very low trapping potentials during the remeasurement sequence and careful control of the size of the injected ion population. Suspended trapping [26] also should be a successful method for elimination of space-charge effects so that high performance remeasurement spectra can be obtained.

\section{Acknowledgments}

This work was supported by the Welch Foundation, the National Institutes of Health, and the National Science Foundation.

\section{References}

1. Henry, K. D.: Quinn, J. P.; McLafferty, F. W. I. Am. Chem. Soc. 1991, 113, 5447-5449.

2. Beu, S. C.; Senko, M. W.; Quinn, J. P.; McLafferty, F. W. I. Am. Soc. Mass Spectrom. 1993, 4, 190-192.
3. Guan, Z. Q.; Hofstadler, S. A.; Laude, D. A., Jr. Anal. Chem. 1993, 65, 1588-1593.

4. Smith, R. D.; Loo, J. A.; Edmonds, C. G.; Barinaga, C. J.; Udseth, H. R. Anal. Chem. 1990, 62, 882-899.

5. Senko, M. W.; Beu, S. C.; McLafferty, F. W. Anal. Chem. 1994, $66,415-417$.

6. Comisarow, M. B. In Ion Cyclotron Resonance Spectrometry II; Springer: New York, 1982; Vol. 2, pp 484-513.

7. Williams, E. R.; Henry, K. D.; McLafferty, F. W. J. Am. Chem. Soc. 1990, 112, 6157-6162.

8. Guan, Z.; Campbell, V. L.; Drader, J. J.; Laude, D. A. Presented at the 42nd ASMS Conference on Mass Spectrometry and Allied Topics, Chicago, IL, 1994; p 1012.

9. Guan, Z.; Drader, J. J.; Campbell, V. L.; Laude, D. A. Anal. Chem. 1995, 67, 1453-1458.

10. Campbell, V. L.; Guan, Z.; Vartanian, V. H.; Laude, D. A. Anal. Chem. 1995, 67, 420-425.

11. Speir, J. P.; Gorman, G. S.; Pitsenberger, C. C.; Turner, C. A.; Wang, P. P.; Amster, I. J. Anal. Chem. 1993, 65, 1746-1752.

12. Hendrickson, C. L.; Laude, D. A. Anal Chem., accepted.

13. Guan, S.; Marshall, A. G. J. Chem. Phys. 1993, 98, 4486-4493.

14. Guan, S.; Wahl, M. C.; Marshall, A. G. J. Chem. Phys. 1994, $100,6137-6140$.

15. Marto, J. A.; Guan, S.; Marshall, A. G. Rapid Commun. Mass Spectrom. 1994, 8, 615-620.

16. Campbell, V. L.; Guan, Z.; Laude, D. A., Jr. Presented at the 41st ASMS Conference on Mass Spectrometry and Allied Topics, San Francisco, CA, 1993; p 443.

17. Guan, Z.; Campbell, V. L.; Laude, D. A. Rev. Sci. Instrum., accepted.

18. Hofstadler, S. A.; Beu, S. C.; Laude, D. A., Jr. Annl. Chem. 1993, 65, 312-316.

19. Hofstadler, S. A.; Laude, D. A., Jr. Int. J. Mass Spectrom. Ion Processes 1990, 101, 65-78.

20. Campbell, V. L.; Guan, Z.; Laude, D. A., Jr. J. Am. Soc. Mass Spectrom. 1994, 5, 221-229.

21. Comisarow, M. B.; Marshall, A. G. Chem. Phys. Lett. 1974, 25, 282-283.

22. Comisarow, M. B.; Marshall, A. G. Chem. Phys. Lett. 1974, 26, 489-490.

23. Ledford, E. B., Jr.; Rempel, D. L.; Gross, M. L. Anal. Chem. 1984, 56, 2744-2748.

24. Jeffries, J. B.; Barlow, S. E.; Dunn, G. H. Int. J. Mass Spectrom. Ion Processes 1983, 54, 169-187.

25. Dunbar, R. C.; Chen. J. H.; Hays, J. D. Int. J. Mass Spectrom. Ion Processes 1984, 57, 39-56.

26. Laude, D. A.; Beu, S. C. Anal. Chem. 1989, 61, 2422-2427. 